\title{
Asymmetric Localization of CK2a During Xenopus Oogenesis
}

\author{
Gregory A. Imbrie, Hao Wu, David C. Seldin and Isabel Dominguez*
}

Hematology-Oncology Section, Department of Medicine, Boston University Medical School, 650 Albany Street, Boston, MA, USA

\begin{abstract}
The establishment of the dorso-ventral axis is a fundamental process that occurs after fertilization. Dorsal axis specification in frogs starts immediately after fertilization, and depends upon activation of $\mathrm{Wnt} / \beta$-catenin signaling. The protein kinase CK2 $\alpha$ can modulate Wnt/ $\beta$-catenin signaling and is necessary for dorsal axis specification in Xenopus laevis. Our previous experiments show that CK2 $\alpha$ transcripts and protein are animally localized in embryos, overlapping the region where $\mathrm{Wnt} / \mathrm{\beta}$-catenin signaling is activated. Here we determined whether the animal localization of CK2 $\alpha$ in the embryo is preceded by its localization in the oocyte. We found that CK2a transcripts were detected from stage I, their levels increased during oogenesis, and were animally localized as early as stage III. CK2 $\alpha$ transcripts were translated during oogenesis and CK2 $\alpha$ protein was localized to the animal hemisphere of stage VI oocytes. We cloned the CK2 $\alpha$ 3'UTR and showed that the $2.8 \mathrm{~kb}$ CK2 $\alpha$ transcript containing the 3'UTR was enriched during oogenesis. By injecting ectopic mRNAs, we demonstrated that both the coding and 3'UTR regions were necessary for proper CK2 $\alpha$ transcript localization. This is the first report showing the involvement of coding and 3'UTR regions in animal transcript localization. Our findings demonstrate the pre-localization of CK2 $\alpha$ transcript and thus, CK2 $\alpha$ protein, in the oocyte. This may help restrict CK2 $\alpha$ expression in preparation for dorsal axis specification.
\end{abstract}

Keywords: CK2 $\alpha$; Animal; Xenopus oocyte; RNA localization; Protein localization; 3'UTR; Coding; Asymmetry

\section{Introduction}

The establishment of the embryonic axes is a fundamental process that dictates the subsequent development of the body plan. In a number of species, the dorsoventral axis is set before the first cleavage through the repositioning of molecules that are asymmetrically distributed during oogenesis [1,2]. In Xenopus laevis, the molecules essential for the development of dorsal structures are called dorsal determinants. The dorsal determinants are found vegetally in oocytes [3-5], and their composition is beginning to be understood [6-8]. Shortly after fertilization, the rotation of the egg cortex relative to the underlying cytoplasm moves the dorsal determinants to the prospective dorsal side of the embryo [5]. This cortical rotation is essential for embryonic development because if inhibited, the embryo does not develop any differentiated structures or embryonic axes [5]. At the end of cortical rotation, the dorsal determinants lie in contact with the equatorial cytoplasm. It is thought that the interaction between the dorsal determinants and the equatorial (marginal) cytoplasm activates the dorsal program [9]. This interaction results in the activation of Wnt/ $\beta$ catenin signaling $[10,11]$, a pathway required for dorsal axis formation in Xenopus laevis [12-14].

A number of components of $\mathrm{Wnt} / \beta$-catenin signaling have been identified to play a role in dorsal axis formation in Xenopus laevis [12]. We recently identified CK2 as a bona fide component of canonical Wnt signaling that is necessary and sufficient for Xenopus laevis dorsal axis formation [15]. CK2 is required for the stabilization of the key component of $\mathrm{Wnt} / \beta$-catenin signaling, $\beta$-catenin [15-18]. Intriguingly, transcript and protein for the catalytic $(\alpha)$ and regulatory $(\beta)$ subunits of CK2 are enriched in animal blastomeres of the Xenopus morula [19] and are present in animal and equatorial blastomeres in the Xenopus blastula $[15,19]$. This precise CK2 localization may be required for proper regulation of $\beta$-catenin, as the distribution of endogenous CK2 [15] overlaps the equatorial region where $\beta$-catenin upregulation occurs in the embryo $[11,20]$.

Asymmetric transcript and protein localization along the animalvegetal axis of the Xenopus embryo is typically maternally derived from the oocyte [21-25]. During Xenopus laevis oogenesis, a number of transcripts are found localized to the vegetal or animal hemispheres. Vegetally enriched transcripts code for proteins that are essential for dorsal axis formation, e.g. dorsal determinants such as XWnt11 [26], germ layer specification, e.g. VegT [27-29], germ cell lineage specification, e.g. Xdazl [30] and cell movements, e.g. Vg1 [31,32]. Two mechanisms have been proposed for vegetal localization of transcripts during oogenesis, an early and a late pathway (reviewed in [33-35]). The early pathway starts at stage I and is responsible for the localization of transcripts involved in germ cell determination. The late pathway starts around stage II and is responsible for the localization of dorsal determinants and mesendodermal determinants. Both pathways require transcript-specific sequences in the 3' untranslated regions (UTR) for proper transcript localization [33-35].

Animally located transcripts in Xenopus laevis are important for embryonic germ layer specification, e.g. POU60/Oct- 60 [36,37], cell signaling, e.g. XWnt5a, [38], and cell polarity, e.g. par-1, [39]. A number of studies suggest a complex regulation of animal localization during Xenopus laevis oogenesis, however no mechanism for animal transcript localization has been described. Animally enriched transcripts may localize gradually throughout oogenesis, e.g. Xbub3 [40] and Xoom [41] or late in oogenesis, e.g. XGß1 [42]. Furthermore, animally enriched transcripts are found localized to the animal subcortical layer, e.g. Ets-1, Ets-2 [43], $X G \beta 1, P A P B P$ [44], to the perinuclear region, e.g. $X b u b 3, P A B P[44]$, or throughout the animal hemisphere, e.g.

*Corresponding author: Isabel Dominguez, Hematology-Oncology Section, Department of Medicine, Boston University Medical School, 650 Albany Street, Boston, MA, USA, Tel. 617-414-1829; Fax: 617- 638-7530; E-mail: isdoming@ bu.edu

Received February 22, 2011; Accepted May 02, 2011; Published May 05, 2012

Citation: Imbrie GA, Wu H, Seldin DC, Dominguez I (2012) Asymmetric Localization of CK2 $\alpha$ During Xenopus Oogenesis . Human Genet Embryol S4:001. doi:10.4172/2161-0436.S4-001

Copyright: (c) 2012 Sofocleous C, et al. This is an open-access article distributed under the terms of the Creative Commons Attribution License, which permits unrestricted use, distribution, and reproduction in any medium, provided the original author and source are credited. 
An3 [31]. In addition, in some cases both protein and transcripts are animally restricted, e.g. Xoom $[41,45]$, in other cases, the protein is animally restricted while the transcripts are ubiquitous, e.g. IP3R [46]. In this study, our goal was to ascertain the dynamics of localization of the catalytic subunit of $\mathrm{CK} 2, \mathrm{CK} 2 \alpha$, during Xenopus laevis oogenesis.

\section{Materials and Methods}

\section{Oocyte collection, culture, treatment and injection}

Frogs were housed and used in accordance with relevant guidelines and regulations and according to a protocol approved by the Boston University Institutional Animal Care and Use Committee.

For oocyte isolation, ovaries surgically obtained from tricaineanesthetized females were subjected to digestion with Liberase Blendzyme (Roche, $1.4 \mathrm{mg} / 10 \mathrm{ml}$ ) in OR2 (1X OR2 is $80 \mu \mathrm{M} \mathrm{NaCl}, 2.5$ $\mu \mathrm{M} \mathrm{KCl}_{2}, 1 \mu \mathrm{M} \mathrm{Na}_{2} \mathrm{HPO}_{4}, 3.8 \mu \mathrm{M} \mathrm{NaOH}, 5 \mu \mathrm{M}$ HEPES, $\mathrm{pH} 7.8$, sterile filtered) for 40 minutes. The oocytes were then washed 10 times with 50 ml $1 X$ Modified Barth's Saline (MBS) without $\mathrm{Ca}^{2+}$ and placed in Petri dishes (50-60 oocytes /per $60 \mathrm{~mm}$ dish) in 1X MBS (1X MBS is $88 \mu \mathrm{M}$ $\mathrm{NaCl}, 1 \mu \mathrm{M} \mathrm{KCl}, 10 \mu \mathrm{M}$ HEPES, $0.8 \mu \mathrm{M} \mathrm{Mg}_{2} \mathrm{SO}_{4}, 0.3 \mu \mathrm{M} \mathrm{Ca}\left(\mathrm{NO}_{3}\right)_{2}, 0.4$ $\mu \mathrm{M} \mathrm{CaCl}, \mathrm{pH} 7.6$ sterile filtered). Where indicated in the text, stage $\mathrm{V}$ and VI oocytes were obtained by manual defolliculation. Oocytes were left to rest overnight before manipulation and were stored and maintained during experiments at $18^{\circ} \mathrm{C}$ in $1 \mathrm{X} \mathrm{MBS}$ and the media was changed twice a day.

Oocytes were sorted into stages I to VI according to Dumont [47] based on features including pigmentation and diameter, and were visualized and photographed with a Leica MZ6 dissecting microscope. Oocytes were injected in $1 \mathrm{X}$ MBS with 5-10 nl of indicated solutions. The site of injection varied depending upon the experiment as indicated in the results section. To obtain mature oocytes, stage VI oocytes were treated in vitro with $1 \mu \mathrm{M}$ progesterone for 5-7 hours until GVBD (Germinal Vesicle Breakdown) was morphologically visible as a white spot in the animal pole of the oocyte. GVBD was confirmed by the absence of nuclei after TCA fixation [48].

\section{Whole mount in situ hybridization (WISH)}

De-folliculated pigmented oocytes were fixed in fresh MEMFA (MOPS 0.1 M pH 7.4, EGTA 2 mM, 1 mM MgSO4, 1.4\% formaldehyde) for 1 hour. After fixation, oocytes were dehydrated with $\mathrm{MeOH}$ in graded steps to $100 \%$ and stored at $-20^{\circ} \mathrm{C}$. In situ hybridization was performed as described in [15]. Hybridized probe appears as purple staining. For photography, oocytes were partially bleached and cleared.

\section{Oocyte bisection for RNA and protein analysis}

Before bisection for RNA isolation, oocytes were lightly fixed to avoid loss of cytoplasmic content. Oocytes at stages III and IV were incubated for 10 minutes in $1 \mathrm{X} \mathrm{MBS} / 10 \%$ methanol. Oocytes at stages V, VI, and matured oocytes were incubated for 5 minutes in $1 \mathrm{X}$ $\mathrm{MBS} / 10 \%$ methanol. Ooocytes were stripped of their vitelline envelope with forceps. In the case of the oocytes, this eliminated the potential contamination of oocytes with any remaining follicle cells. Oocytes were then laid on their side and cut into animal and vegetal halves with a steel knife. During cutting, the oocyte halves seal shut and the cytoplasmic contents are preserved. Halves were snap frozen on dry-ice and stored at $-80^{\circ} \mathrm{C}$.

For protein analysis, oocytes were fixed in MEMFA for 18 minutes based on the study of [11]. After fixation, oocytes were washed three times in $1 \mathrm{X}$ MBS. Oocytes were stripped of their vitelline envelope and cut into animal and vegetal halves and frozen as described above. During cutting, the halves remain open however there is no cytoplasmic loss. Halves were snap frozen on dry-ice and stored at $-80^{\circ} \mathrm{C}$.

\section{RNA isolation and analysis of transcript copy number}

For RNA isolation, pools of five (oocytes at stages V or VI) or ten (oocytes at stage III and IV) halves were processed. RNA was extracted with Trizol $^{\circledR}$ (Invitrogen), DNAse I treated, and cDNA was prepared from $1 \mu \mathrm{g}$ total RNA using the BioRad iScript cDNA Synthesis Kit according to manufacturer's instructions. Priming was via random and oligo-dT priming. Control "-RT" samples were made with no reverse transcriptase in the reaction. Quantitative PCR (qPCR) was carried out in a $25 \mu \mathrm{l}$ iTaq Sybr Green reaction (BioRad), in the presence of 400 $\mathrm{nM}$ of each primer in a Stratagene mx3000P real-time PCR machine. Samples were analyzed in duplicate. It was determined for each sample and copy number was determined using a standard formula: $10^{(\mathrm{Ct}-40) /}$ ${ }^{3.32)}$. Transcript copy number was normalized to the copy number for ornithine decarboxylase (Odc).

Sequences of primers for qPCR were chosen using the Primer Express primer analysis program and each primer set was verified by performing qPCR amplification with the primers on cellular cDNA and plasmid DNA, if applicable. Primers with distinct dissociation curve peaks and linear quantitation of cDNA (over 10 two-fold dilutions) and plasmid DNA (over 10 two-fold dilutions) were used for experimental qPCR analysis. When possible, primers were chosen that spanned an intron. The following sequences were used for $\mathrm{qPCR}$ analysis. CK2 $\alpha$ : (forward: 5' AAAGATCCTGGAGAACCTGCG 3'; reverse: 5' TGTTCGAAGACAAGTGCTGGC 3'); 3'UTR of CK2 $\alpha$ : (forward: 5' ATGAGCCTGATGCCCCATATC 3'; reverse: 5' ACACATTCCATCAGTGCACCC 3'); XWnt5a: (forward: 5' GGTTTGCCAAGGAGTTTGTCG 3'; reverse: 5' TCCGGCCTCATTATTGTGGAG 3'); XWnt11: (forward: 5' AGGACAGGCTGTGCAACAAGA 3'; reverse: 5' TCCACGATGGTTTCGGTGTAG 3'); and GFP: (forward: 5' TAAACGGCCACAAGTTCAGCG 3'; reverse: 5' CGGTGGTGCAGATGAACTTCA 3').

\section{Protein isolation and immunoblot analysis}

For protein analysis, pools of 10 halves of stage VI oocytes were homogenized in 1X Laemmli buffer. Proteins were electrophoresed and electroblotted onto polyvinylidene difluoride (PVDF) membranes (Millipore) and quantitative immunoblotting analysis using Fluor-S MultiImager (BioRad) was performed as described in [15]. The volume equivalent of 0.2 stage VI oocytes (stage VI volume $=1 \mu$ l) was loaded for immunoblotting, as it was within the linear range of detection for all proteins studied (I. Dominguez, unpublished data).

\section{Xenopus CK2a 3'UTR Cloning}

From a discontinuous megablast (NCBI database) to the mouse 3'UTR (G.A. Imbrie, unpublished data), an EST clone containing CK2a was identified: IMAGE ID: 4971090 (Genbank Accession \#: CF289385/ CF289386). This clone was sequenced. By homology alignment, it was found to contain the full-length CK2 $\alpha$ gene including a 960bp 3'UTR, extending from base 1395 to 2356 . The 3'UTR contains a polyadenylation signal. This clone was termed pCMV-Sport6-CK2a-3'UTR. The 960bp 3' UTR fragment was isolated by PCR with Elongase, using a forward primer that introduced an XbaI site (GCATTCTAGATAGGAGCCATCACAGTTGACC) and a reverse primer that introduced a NotI site (GCATGCGGCCGCGATAAATAAAACCATGTTTATTTACACC). 
Citation: Imbrie GA, Wu H, Seldin DC, Dominguez I (2012) Asymmetric Localization of CK2a During Xenopus Oogenesis . Human Genet Embryol S4:001. doi:10.4172/2161-0436.S4-001

Page 3 of 9

\section{Northern blot analysis}

The Xenopus CK2 a probe was generated by MluI and NdeI restriction digestion of the pCMV-Sport6-CK2 $\alpha$-3'UTR plasmid which contained the CK2 $\alpha$-UTR clone, producing a 2060bp fragment containing the CK $2 \alpha$ coding sequence, and $684 \mathrm{bp}$ of the $3^{\prime} \mathrm{UTR}$. The digestion also produced a 296bp fragment of the most 3' region of the 3'UTR; these fragments were isolated by gel extraction. Probes were labeled with $\alpha^{32} \mathrm{P}$ dCTP using Klenow, purified using a ProbeQuant G-50 Micro Column (Amersham Biosciences) and the percent incorporation was measured in a scintillation counter. Northern blots were hybridized with radioactive labeled probe using Stratagene QuikHyb Solution (catalog \#201220) following the manufacturer's recommended protocol. The transcripts detected on the Northern membranes were quantified in a phosphoimager (Bio-Rad GS505 Exposure platform, Molecular Imaging Screen-BI and GS-525 Molecular Imager System reader and analyzed with Multi-Analyst software).

\section{Generation of eGFP constructs}

To generate pCS2-EGFP and pCS2-EGFP-CK2 $\alpha$, the GFP coding sequence was amplified by PCR from plasmid pEGFP-C1 with a forward primer containing a ClaI site (GGA TTC ATC GAT ATG GTG AGC AAG GG) and a reverse primer containing StuI and EcoRI sites (AGGCCT GAA TTC CTT GTA CAG CTC GTC). The product was ligated into the pCS2-CK2 $\alpha$ plasmid [15] or pCS2 alone to form pCS2-EGFP-CK2 $\alpha$ and pCS2-EGFP, respectively.

To generate pCS2-EGFP-CK2-3'UTR and pCS2-EGFP-3'UTR, the 3'UTR was amplified by PCR and the plasmids pCS2-EGFP and pCS2-EGFP-CK2 $\alpha$ were digested with XbaI and NotI. This digestion removed the SV40 polyadenylation signal present in the $\mathrm{pCS} 2$ vector. The 3'UTR fragment was ligated into the linearized pCS2-EGFP and pCS2-EGFP-CK2 $\alpha$ plasmids with Quick Ligase to form pCS2-EGFPCK2-3'UTR and pCS2-EGFP-3'UTR.

\section{Results}

CK2 $\alpha$ transcript levels and dynamic localization during oogenesis

CK2 $\alpha$ transcripts are enriched in the animal hemisphere of stage 3 embryos by whole mount in situ hybridization (WISH) [15] and realtime PCR (RT-qPCR, not shown). Since molecules with asymmetrical distribution in the early embryo typically inherit their patterns from the oocyte, we examined the levels and localization of CK2 $\alpha$ transcripts during oogenesis. RT-qPCR analysis showed CK2 $\alpha$ transcripts present and increasing in levels during oogenesis (Figure 1A). Next, we determined whether and when CK2 $\alpha$ transcripts are animally distributed in the Xenopus laevis oocyte by WISH. As with RT-qPCR analysis, CK2 $\alpha$ transcripts were detected throughout oogenesis. Enriched animal hemisphere staining was first observed in stage III oocytes, and clear animal hemisphere staining was found in stage IV and V oocytes (Figure 1B). In stage VI, staining was further restricted by exclusion from the animal cortex and subcortical cytoplasm. Stage VI oocytes matured in vitro showed similar staining to stage VI oocytes, like other animally localized transcripts that remain animally localized following oocyte maturation [22] (not shown). No-probe controls did not show staining (Figure 1C). These findings suggest that animal restriction of CK2 $\alpha$ transcripts occurs in early oogenesis.

As WISH sometimes fails to reveal vegetally located transcripts due to poor probe penetration through the yolk, we analyzed the localization of CK $2 \alpha$ transcripts by RT-qPCR. Late stage III and stage
$\mathrm{V}$ oocytes were divided into animal and vegetal portions as shown in figure S1A. As controls we examined XWnt11 and XWnt5a transcripts (Figure $2 \mathrm{~A}, \mathrm{~B}$ ). $O d c$ was used as a control as we found more evenly

A

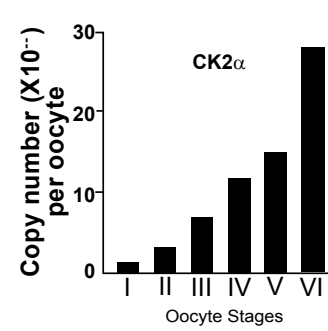

C

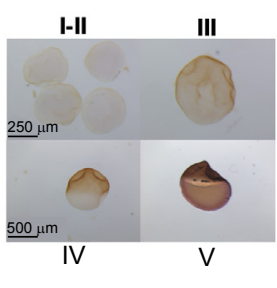

B
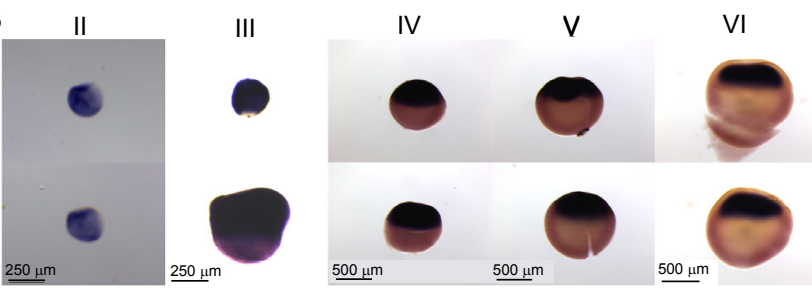

Figure 1: CK2 $\alpha$ transcripts levels and localization by WISH

(A) Analysis of total CK2 $\alpha$ transcripts in Xenopus oocytes by RT-qPCR Oocytes were collected, RNA extracted and quantitative PCR was carried out with specific primers to the CK2 $\alpha$ coding sequence. The transcript for CK2 $\alpha$ increases during oogenesis. This experiment was repeated twice with similar results.

(B,C) Whole mount in situ hybridization of oocytes at different stages with an antisense CK2 $\alpha$-digoxigenin-labeled probe. (B) Chromogenic staining, shown in purple, indicates transcript localization. (C) Representative no probe controls for the in situ hybridizations. Animal hemisphere is up in all panels. Scale bars $250 \mu \mathrm{m}$ or $500 \mu \mathrm{m}$ as indicated in picture. This experiment was repeated using oocytes from three different frogs with similar results.
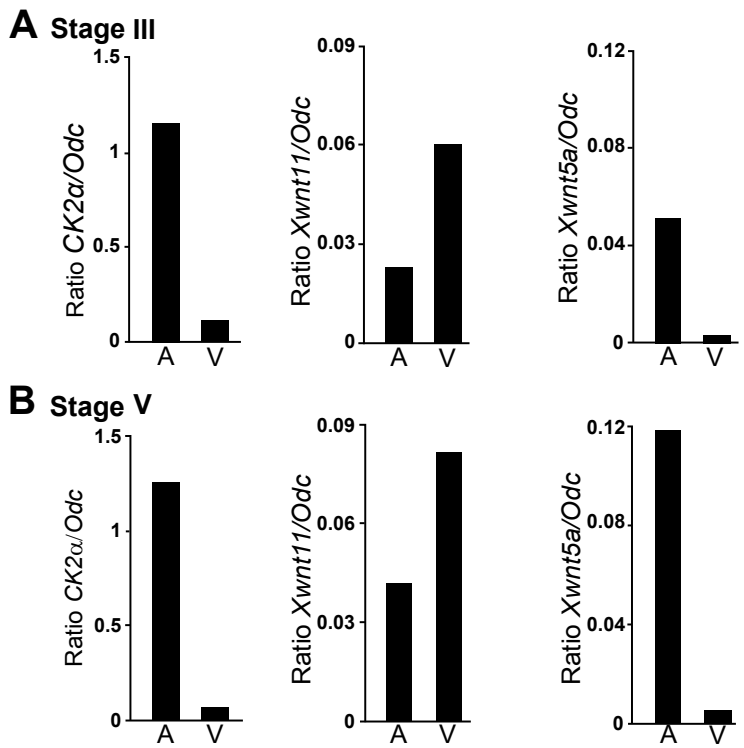

Figure 2: CK2 $\alpha$ transcript localization by RT-qPCR.

RT-qPCR analysis of 10 pooled animal (A) or vegetal $(\mathrm{V})$ halves at late stage III (A) and stage $V(B)$ stages of oogenesis with specific primers for CK2 $\alpha$ $X W n t 11$, a known vegetally localized transcript; $X W n t 5$, an animally enriched transcript. From left to right, histograms represent transcript number normalized by Odc for CK2a, XWnt11 and XWnt5a. This experiment was repeated three times for CK2 $\alpha$ and Odc, and twice for Wnts with similar results. 
distributed throughout the oocyte than Gapdh [22]. Consistent with reported results, $X W n t 5 a$ localized to the animal portion of the oocyte [38]. XWnt11 transcripts were present both animally and vegetally, with enrichment in the vegetal hemisphere at stage III, in agreement with in situ hybridization analysis [26]. At stages V and VI, even though in situ hybridization shows exclusive vegetal localization [26], by RT-qPCR, XWnt11 transcripts can also be found in the animal hemisphere and equatorially $[22,49]$. RT-qPCR analysis demonstrated that $\mathrm{CK} 2 \alpha$ transcripts were preferentially localized animally at late stage III (Figure 2A), stage V (Figure 2B), stage VI and matured oocytes (not shown). Thus, CK2 a transcripts localize to the animal hemisphere during oogenesis as early as stage III.

\section{CK2 $\alpha$ protein is localized animally in oocytes}

$\mathrm{CK} 2 \alpha$ protein was present at all stages of oogenesis and increased overtime indicating that the transcript is continuously translated (Figure 3A). In contrast, the transcript for another serine threonine kinase GSK3 $\beta$ decreased during oogenesis compared to tubulin (Figure $3 \mathrm{~A})$. Using these data, we calculated the protein content per oocyte. CK2 $\alpha$ and tubulin increase approximately 125 fold from stage I to stage VI, what is proportional to the increase in oocyte volume (Figure 3B).

During Xenopus oogenesis asymmetrically distributed proteins can be found along the animal-vegetal axis of the oocyte [23,24,50,51]. We examined the animal/vegetal distribution of CK2 $\alpha$ protein in stage VI oocytes by immunoblot of animal and vegetal halves obtained by cutting oocytes along the pigmentation line (Figure S1B). Immunoblot analysis showed that CK2 $\alpha$ was localized in the animal half of the stage VI oocyte while XGSK $3 \beta$ was detected in both animal and vegetal halves (Figure $3 \mathrm{C}$ ). Thus, CK2 $\alpha$ protein localizes to the animal hemisphere of full-grown stage VI oocytes.

\section{CK2 $\alpha$ transcripts in oocytes and cloning of CK2 2 3'UTR}

We analyzed CK2 $\alpha$ transcript size during oogenesis by Northern blot using a CK2 $\alpha$ probe. Two major CK2 $\alpha$ transcripts of approximately $2.8 \mathrm{~kb}$ and $1.8 \mathrm{~kb}$ were detected (Figure $4 \mathrm{~A}$ ), similarly to those reported in adult tissues [52]. The ratio of $2.8 \mathrm{~kb}$ and $1.8 \mathrm{~kb}$ CK2 $\alpha$ transcripts increased 3-4 fold from stage I to stage VI oocytes (Figure 4B).

The $2.8 \mathrm{~kb}$ transcript was identified as containing the 3'UTR by probing a Northern blot with a 296 pb fragment of the CK2 $\alpha$ 3'UTR (X3'UTR) (Figure 4A). For this, we identified the CK2 $\alpha$ EST clone containing the $3^{\prime} \mathrm{UTR}$ from a discontinuous megablast (NCBI database) to the mouse 3'UTR (Figure 4C). We obtained the Xenopus clone (CF289385/CF289386) from the IMAGE consortium and used it to isolate the $960 \mathrm{bp} \mathrm{3'UTR} \mathrm{sequence} \mathrm{used} \mathrm{as} \mathrm{a} \mathrm{northern} \mathrm{probe} \mathrm{(X3'UTR).}$ Since the $2.8 \mathrm{~kb}$ transcript is favored during oogenesis, this supports the idea that the 3'UTR may be playing a role in CK2 $\alpha$ function during oogenesis.

\section{Coding and 3'UTR regions are necessary for localization}

In Xenopus, injected ectopic mRNAs containing the 3'UTR localize in a similar pattern to their endogenous counterparts [53,54]. We used the same strategy to determine whether the 3'UTR of CK2 $\alpha$ plays a role in animal localization of CK2 $\alpha$ transcripts. For this, three GFPexpressing plasmid constructs were engineered (Figure 5A): one containing only the CK2 $\alpha$ 3'UTR (GFP-3'UTR); one containing the CK2 $\alpha$ coding region alone (GFP-CK2 $\alpha$ ); and one containing both (GFP-CK2 $\alpha-3$ 'UTR). The SV40 polyadenylation sequence present in the original plasmid was removed during the generation of the GFP3'UTR and GFP-CK2 $\alpha-3$ 'UTR plasmids. mRNAs were transcribed in vitro from the three plasmids and injected into the vegetal hemisphere of stage IV oocytes. After 48 hours, the animal/vegetal (A/V) ratio of the mRNAs was calculated to assess ectopic transcript relocalization by RT-qPCR to the GFP sequence. mRNAs that relocalize to the animal hemisphere would have an $\mathrm{A} / \mathrm{V}$ ratio of more than one, while mRNAs that do not relocalize would have an $\mathrm{A} / \mathrm{V}$ ratio less than one. By 48 hours, the GFP-CK2 $\alpha-3$ 'UTR mRNA had an A/V ratio of 1.45 , suggesting that
A

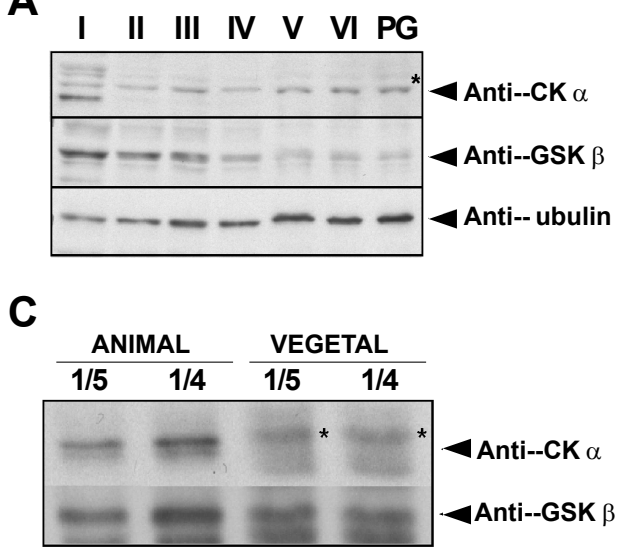

B

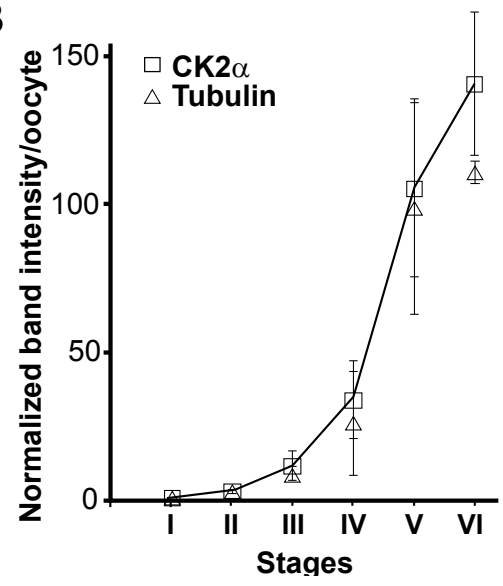

Figure 3: CK2 $\alpha$ protein is enriched in the animal hemisphere of stage $\mathrm{VI}$ Xenopus oocytes

(A) Expression of CK2 proteins during Xenopus oogenesis. Extracts were prepared from oocytes stages I, II, III, IV, V, VI, and stage VI treated with progesterone (PG). The volume equivalent of 0.2 stage VI oocytes was subjected to immunoblotting. Thus, we loaded, 22 stage I oocytes, 6.8 stage II oocytes, 2.5 stage III oocytes, 0.7 stage IV oocytes, 0.3 stage $\mathrm{V}$ oocytes and 0.2 stage $\mathrm{VI}$ oocytes. All immunoblots were repeated three times. The asterisk (*) indicates previously identified non-specific cross-reactive proteins in Xenopus extracts [15].

(B) Graph representing the data (band intensity) from the three independent experiments as in Figure 3A. Protein band intensity was divided by the number of oocytes loaded to obtain the band intensitity per oocyte, normalized to stage I oocyte (stage I =1), and represented as mean \pm S.D.

(C) Immunoblot analysis of CK2 protein levels in 10 pooled stage VI animal or vegetal halves. One fifth $(1 / 5,0.2 \mu \mathrm{l})$ and one fourth $(1 / 4,0.25 \mu \mathrm{l})$ of oocyte lysate were loaded for immunoblot analysis. The asterisk $\left(^{*}\right)$ indicates a non-specific cross-reactive protein in Xenopus extracts [15]. CK2 $\alpha$ protein is animally enriched at stage $\mathrm{VI}$ of oogenesis, while other tested proteins are not. This is a representative experiment out of three with identical results. 
Citation: Imbrie GA, Wu H, Seldin DC, Dominguez I (2012) Asymmetric Localization of CK2a During Xenopus Oogenesis . Human Genet Embryol S4:001. doi:10.4172/2161-0436.S4-001

Page 5 of 9

it was relocalized to the animal hemisphere (Figure 5B, left panel). In contrast, the GFP-CK2 $\alpha$ mRNA lacking the 3'UTR had an A/V ratio of 0.65 , suggesting that it was not competent for relocalization (Figure $5 \mathrm{~B}$, left panel). The GFP-3'UTR mRNA had an A/V ratio of 0.4 , also indicating that these mRNAs remained in the vegetal portion of the oocyte (Figure 5B, left panel). These results are summarized in Figure 5A (right panel). The GFP-CK2 $\alpha-3$ 'UTR mRNA also relocalized animally in stage III oocytes 24 hours after injection (Figure 5B, right panel). We corroborated the animal relocalization of GFP-CK2 $\alpha$ 3'UTR mRNA by injecting labelled mRNAs. For this, digoxigeninlabelled mRNAs were injected vegetally into stage IV oocytes and cultured for 24 hours and whole-mount immunohistochemistry with and anti-digoxigenin antibody performed. mRNA transcribed from the GFP-XCK2 $\alpha$ plasmid served as a control. Figure 5C shows that the GFP-CK2 $\alpha-3$ 'UTR mRNA relocalized to the animal hemisphere while the GFP-CK2 $\alpha$ mRNA did not relocalize from its injected position in the vegetal hemisphere. These findings indicate that both the coding region and the 3'UTR are required for proper animal localization of CK2 $\alpha$ transcripts.

\section{Discussion}

Asymmetric localization of mRNA and proteins plays an important role in embryonic development; e.g. in the establishment of the body axes, specification of the germ layers and germ cell lineage, and the control of cellular processes such as cell fate determination, cell polarity and cell movement [13,55-63]. Our understanding of the mechanism of dorsal axis formation is limited because of an incomplete understanding of the nature, distribution and activation of the factors that regulate dorsal axis formation [12-14]. Dorsal axis formation in Xenopus laevis embryos depends upon asymmetric stabilization of $\beta$-catenin, the transcriptional co-activator of the canonical Wnt
A

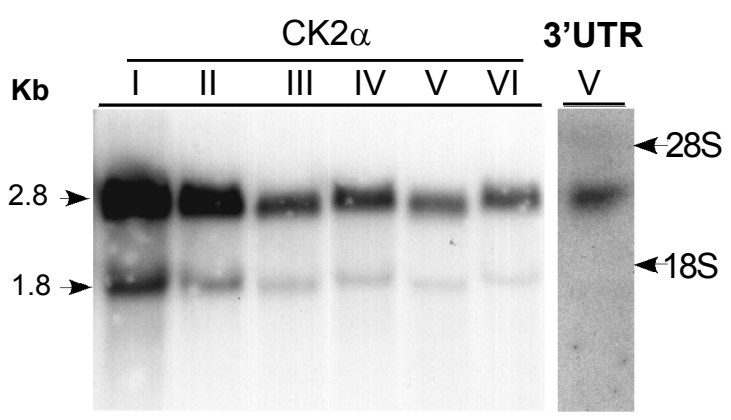

\section{Xenopus laevis CK2alpha 3'UTR}

1 gagccatcac agttgacccc ttggectatc tccctaatt acatcccacc

51 tgatgtggaa ggaggggggc ccccagatat tggaagcccc ctagacacac

101 cgtgtctgag cagttgcttg tggatttata gtagttctgt cattataaat

151 atatatatat aaatataaa aatattatag gctgaataat ttttttact

201 ttgttatagc tcttggtcac aagctgctct tgtgtctagg atgtggtcac

251 tggccccagg ctgccettt gttcctggga tgtggctact ggccagatgt

301 acccacagag gattctttt ttgattgttt ttttttatt tcaggttcac

351 atgagcctga tgccccatat ctcattgtac ccaagtgctg ccaaagccat

401 gatctctctc tgccaccccc tttatggcag acacgagtgg tttgggtgca

451 ctgatggaat gtgtgctgcc ctttttaccc caaccaaagc catgtccgtg

501 tgggatgagc aatacagttt cttttgtaaa cattgcagtg agatctgcat

551 ggtggttcaa gcagacgttc ttctctttac cccctgccet aatgtgccat

601 tctgggaggg attagacatc ttattactat cccagaaatg cctccccaac

651 cccttcgccc tctttaal atgagctgtc gccatatgtc cctgtgaaat

701 cctacctctt tccctgctgg gaagtggccg ttgaggttac caccacctta

751 atcatgttca tgggcaagac ctgctctgat gtctcctatc tctccaccac

801 cctattgcac ccaccccatc ttcccccatt ttaatttgca acgcctgtcc

851 cccagcaagg agctgacgcg gtaacacaaa aaatgcttt tctccttcac

901 atgtgacaga tctaatgttg gaactatttg gtgtaataa acatggtttt

951 atttatcaaa aaaaaaaa aaa

Figure 4: Analysis of CK2 $\alpha$ transcripts during oogenesis and sequence of the XCK2 $\alpha$ 'UTR

(A) Northern blot analysis of RNA extracted from different stages of Xenopus laevis oogenesis using a CK2 $\alpha$ coding sequence probe (CK2 $\alpha$, left columns) or probe specific to the CK2 $\alpha$ 3'UTR (3'UTR, right column). Analysis shows two major CK2 $\alpha$ transcripts, estimated at $2.8 \mathrm{~kb}$ and $1.8 \mathrm{~kb}$. The upper band was identified as containing the 3'UTR sequence. Position of the $28 \mathrm{~S}$ and $18 \mathrm{~S}$ rRNA bands is marked. The northern blots were performed twice with similar results. As younger oocytes express less RNA than late oocytes, we loaded RNA from 2.5 stage I oocytes, 1.3 stage II oocytes, 0.52 stage oocytes III, 0.08 stage IV oocytes, 0.03 stage $\mathrm{V}$ oocytes and 0.01 stage $\mathrm{VI}$ oocytes.

(B) Graph representing the ratio of the $2.8 \mathrm{~kb}$ to $1.8 \mathrm{~kb}$ XCK2 $\alpha$ transcripts quantified from the Northern blot in Figure 4B.

(C) An EST clone containing a XCK2 $\alpha$ and its 3'UTR was identified (IMAGE ID: 4971090, BC072167 / Genbank Accession \#: CF289385/CF289386) and sequenced. The 3'UTR was defined as starting at the first base downstream of the stop codon. 
signaling pathway. Our previous data showed that CK2 can regulate $\beta$-catenin protein stability, and it is an essential regulator of dorsal axis formation in Xenopus laevis embryos [15-17]. CK $2 \alpha / \beta$ is present in the right place, the equatorial region of the embryo, and at the right time to regulate $\beta$-catenin $[15,19]$. Since $C K 2 \alpha / \beta$ proteins and transcripts are animally localized in the embryo, the primary goal of this study was to determine whether CK2 protein and transcript localization is derived maternally. For this, we studied CK2 $\alpha$ as it is the catalytically active protein subunit.

The experiments presented here show that animal hemisphere enrichment of CK2 $\alpha$ transcripts occurs as early as stage III of oogenesis and it is maintained until stage VI. Animal localization in early oogenesis as has been also described for Xbub3 [40] and Xoom [41] while others, such as XG $\beta 1$ [42], localize late in oogenesis. This suggests that, like vegetally localized transcripts, there may be early and late pathways for transcript localization. Our data shows that CK2a transcript localization during oogenesis can explain the transcript distribution in early Xenopus laevis embryos. Interestingly, at stage VI, CK $2 a$ transcript is excluded from the cortex and subcortical cytoplasm, potentially to ensure that $\mathrm{CK} 2 \alpha$ distribution will not be altered due to cortical rotation after fertilization. However, during early embryogenesis, CK2 $\alpha$ transcripts are also present in the most cortical part of the embryo, albeit in a lesser amount than in the inner cytoplasm [15], showing that redistribution of CK2 $\alpha$ transcripts happens after cortical rotation.

CK2 $\alpha$ protein was animally located suggesting that pretranslational localization of the transcript may establish the asymmetric distribution of CK2 $\alpha$ protein. Of note, animal transcript localization does not preclude a contribution of post-translational localization of the proteins, as in the case of XaPKC, ASIP/PIR-3 [64]. CK2 $\alpha$ protein
A
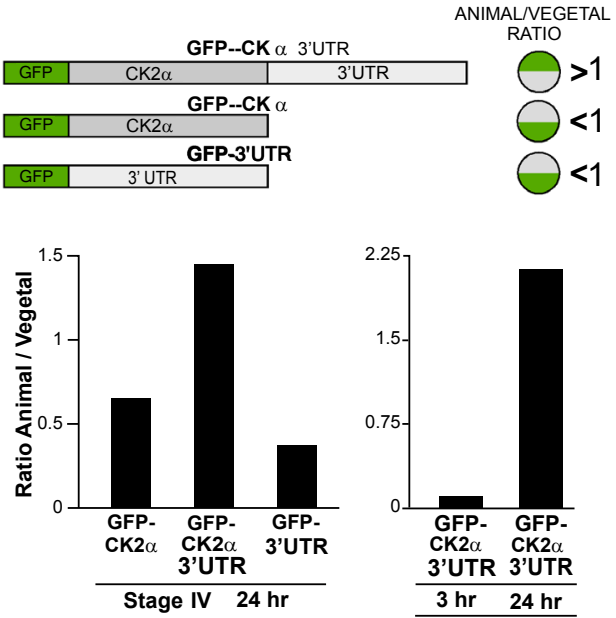

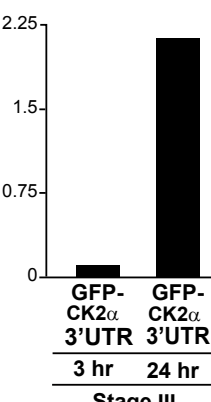

Staqe III

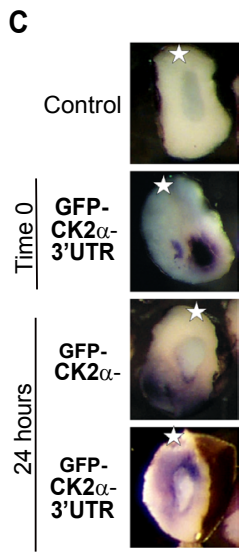

Figure 5: CK2 $\alpha$ coding and 3'UTR transcript regions are necessary for ectopic mRNA localization

(A) On the left, schematic representation of the constructs GFP-CK2 $\alpha-3$ 'UTR, GFP-CK2 $\alpha$ and GFP-3'UTR. On the right, summary of the results presented in this figure.

(B) Histograms depicting the animal to vegetal ratio of ectopic mRNAs. Oocytes were injected vegetally with $1 \mathrm{ng}$ of in vitro transcribed mRNA from the constructs GFP-CK2 $\alpha-3$ 'UTR, GFP-CK2 $\alpha$ and GFP-3'UTR and five animal and vegetal halves were processed for RT-qPCR to the GFP sequence. The animal to vegetal ratio of GFP copy number was calculated (Left histogram) 48 hrs after injection of mRNAs from all the constructs into stage IV, and (Right histogram) 3 and 24 hrs after injection of GFP-CK2 $\alpha-3$ 'UTR mRNA into stage III. The CK2 $\alpha-3$ 'UTR mRNA relocalized animally while CK2 $\alpha$ and 3'UTR mRNAs did not relocalize. These experiments were repeated twice with similar results.

(C) Localization of digoxin-labeled ectopic mRNAs twenty-four hours after vegetal injection. Stage IV oocytes were injected vegetally with 1 ng of in vitro transcribed digoxigenin-labeled sense mRNA from the GFP-CK2 $\alpha-3$ 'UTR and GFP- CK2 $\alpha$ constructs (time 0). 24 hours after injection, oocytes were fixed, stained with antidigoxigenin antibodies as whole-mounts, and bisected with a steel knife for photomicrography. Chromogenic staining is shown in purple. Uninjected oocytes showed no staining (control). ${ }^{*}=$ animal. The CK2 $\alpha-3$ 'UTR mRNA relocalized animally while CK2 $\alpha$ mRNA did not relocalize. This experiment was repeated twice with similar results.

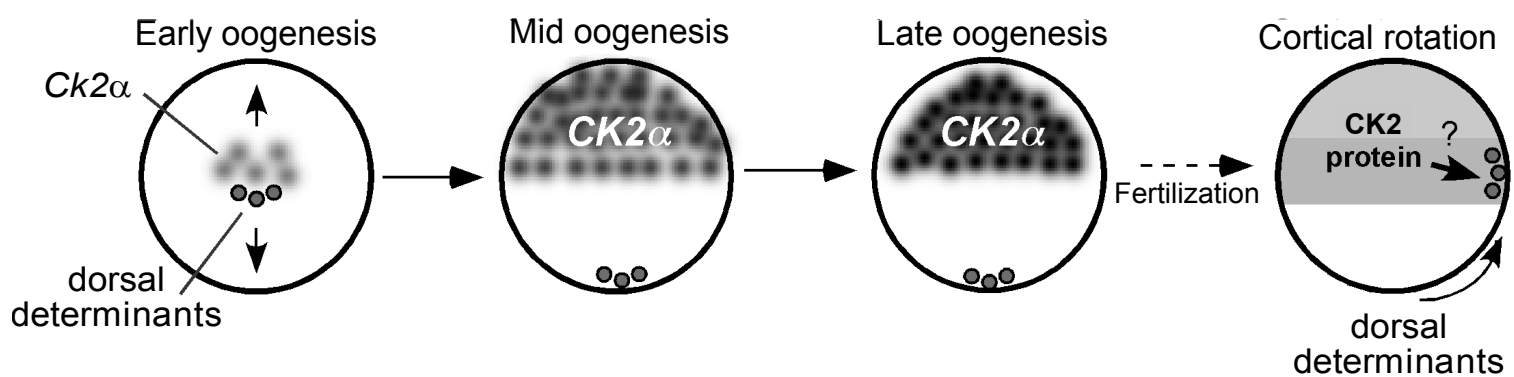

Figure 6: CK2 $\alpha$ localization and dorsal axis formation. During oogenesis, CK2 $\alpha$ mRNA is localized to the animal pole, in a process dependent on its coding and 3'UTR sequences, while the dorsal determinants are localized to the vegetal pole. Fertilized embryos will inherit the dorsal determinants and CK2 protein in nonoverlapping regions that will come together after cortical rotation in the region where dorsal specification will be initiated. 
levels increase during oogenesis corresponding to the reported increase in total CK2 activity during oogenesis [52]. The magnitude of change of CK2 $\alpha$ protein and transcript levels during oogenesis differ, suggesting that CK2 $\alpha$ may be regulated through translational or posttranslational mechanisms that could include phosphorylation by CK2 $\alpha$ [65].

Additionally, we showed an enrichment of the $2.8 \mathrm{~kb}$ CK2 $a$ transcript containing the 3'UTR during oogenesis, correlating with increased animal localization of the transcript. A similar shift in CK2 $\alpha$ transcripts ratio was observed in quiescent versus proliferating mouse cells (G. A. Imbrie, unpublished data) suggesting that the ratio between the transcripts may play a role in the regulation of CK2 $\alpha$ in diverse systems, including other animal models.

Our data shows that $\mathrm{CK} 2 \alpha$ transcript localization requires the coding and 3'UTR regions, which is a novel mechanism for RNA localization in Xenopus oocytes, as vegetal transcript localization depends exclusively on 3'UTR sequences [66]. Although this mechanism is novel for Xenopus, recent work identifies additional regions involved in RNA localization in other models. For example, the 5'UTR for gurken localization in Drosophila oocytes and Glutelin in Oryza sativa; and the coding region for ASH1 localization at the budding tip in Saccharomyces cerevisiae and the $10-k D a \delta$-zein RNA in Oryza sativa [67-69]. Since transcripts and/or proteins for other regulators of dorsal axis specification, such as $C K 2 \beta, X G S K 3 \beta$, dishevelled, Apc, axin, Tcf3 and $\beta$-catenin are, like CK2 $\alpha$, animally restricted [15,22,49], it will be interesting to test whether their localization also depends upon coding and 3'UTR sequences. Given the diversity in the timing and distribution patterns of animally localized transcripts and proteins, it is possible that diverse mechanisms may be used for their localization in the Xenopus oocyte. For example, Ets-1, Ets-2 localize subcortically [43], Xbub3 localizes perinuclearly [40], and some mRNAs and proteins localize dynamically during oogenesis between cellular regions, e.g. PABP [44]. Based on the importance of animally located transcripts in embryonic development, it will be important to determine the molecular and biochemical mechanism(s) of animal localization (e.g. the RNA sequences required for localization, the role of RNA-binding proteins, the dependence on active transport or anchoring to cellular structures and the contribution of the proposed transcript degradation in the vegetal hemisphere [70]).

In summary, we found that $\mathrm{CK} 2 \alpha$ transcript and protein are localized to the animal hemisphere during Xenopus laevis oogenesis. These data are consistent with a model (Figure 6) in which maternal localization ensures that $\mathrm{CK} 2 \alpha$ is localized animally while the dorsal determinants are located vegetally. Cortical rotation after fertilization relocalizes the dorsal determinants to the dorsal equatorial region of the embryo, overlapping the region where CK2 proteins are enriched. CK2 will act on the dorsal determinants to upregulate $\beta$-catenin and initiate dorsal axis formation. Although many questions remain to be answered, our results on CK2 $\alpha$ demonstrates the importance for both the coding and 3'UTR sequences for localization, and therefore may serve as a model for the study of other animally distributed transcripts in Xenopus laevis and other model organisms.

\section{Acknowledgements}

We thank J. Cha and P. Hogan for frog husbandry and T. H. Khan for assistance with figures. We thank M. Liang for training on RT-qPCR analysis. We thank K Symes, T. Holowacz, M. Hlavacova, K. Chea, I. Roman and M. Fox for critically reading the manuscript. We thank members of the $D$. C. Seldin's laboratory and the Developmental Interest Group for helpful discussions. Supported by AHA Grant in Aid 10GRNT3010038 (I.D.), NIGMS 1R01GM098367 (I.D.), a Pilot research grant from the Department of Medicine (I.D.), NIH R01 CA71796 (D.C.S.) and NIEHS P01 ES11624.

\section{References}

1. Prodon F, Pruliere G, Chenevert J, Sardet C (2004) Establishment and expression of embryonic axes: comparisons between different mode organisms. Med Sci 20: 526-538.

2. Eyal-Giladi H (1997) Establishment of the axis in chordates: facts and speculations. Development 124: 2285-2296.

3. Holowacz T, Elinson RP (1993) Cortical cytoplasm, which induces dorsal axis formation in Xenopus, is inactivated by UV irradiation of the oocyte. Development 119: 277-285

4. Fujisue M, Kobayakawa Y, Yamana K (1993) Ocurrence of dorsal axis-inducing activity around the vegetal pole of an uncleaved Xenopus egg and displacement to the equatorial region by cortical rotation. Development 118: 163-170.

5. Elinson RP, Holowacz T (1995) Specifying the dorsoanterior axis in frogs: 70 years since Spemann and Mangold. Curr Top Dev Biol 30: 253-285.

6. Miller JR, Rowning BA, Larabell CA, Yang-Snyder JA, Bates RL, et al. (1999) Establishment of the dorsal-ventral axis in Xenopus embryos coincides with the dorsal enrichment of dishevelled that is dependent on cortical rotation. J Cell Biol 146: 427-437.

7. Tao Q, Yokota C, Puck H, Kofron M, Birsoy B, et al. (2005) Maternal wnt11 activates the canonical wnt signaling pathway required for axis formation in Xenopus embryos. Cell 120: 857-871.

8. Weaver C, Farr GH, Pan W, Rowning BA, Wang J, et al. (2003) GBP binds kinesin light chain and translocates during cortical rotation in Xenopus eggs. Development 130: 5425-5436.

9. Kageura H (1997) Activation of dorsal development by contact between the cortical dorsal determinant and the equatorial core cytoplasm in eggs of Xenopus laevis. Development 124: 1543-1551.

10. Marikawa Y, Li Y, Elinson RP (1997) Dorsal determinants in the Xenopus egg are firmly associated with the vegetal cortex and behave like activators of the Wnt pathway. Dev Biol 191: 69-79.

11. Larabell CA, Torres M, Rowning BA, Yost C, Miller JR, et al. (1997) Establishment of the dorso-ventral axis in Xenopus embryos is presaged by early asymmetries in beta-catenin that are modulated by the Wnt signaling pathway. J Cell Biol 136: 1123-1136.

12. Weaver C, Kimelman D (2004) Move it or lose it: axis specification in Xenopus Development 131: 3491-3499.

13. Croce JC, McClay DR (2006) The canonical Wnt pathway in embryonic axis polarity. Semin Cell Dev Biol 17: 168-174.

14. Marikawa Y, Elinson RP (1999) Relationship of vegetal cortical dorsal factors in the Xenopus egg with the Wnt/beta-catenin signaling pathway. Mech Dev 89: 93-102.

15. Dominguez I, Mizuno J, Wu H, Song DH, Symes K, et al. (2004) Protein kinase CK2 is required for dorsal axis formation in Xenopus embryos. Dev Biol 274 110-124.

16. Song DH, Sussman DJ, Seldin DC (2000) Endogenous protein kinase CK2 participates in Wnt Signaling in mammary epithelial cells. J Biol Chem 275 23790-23797.

17. Song DH, Dominguez I, Mizuno J, Kaut M, Mohr SC, et al. (2003) CK2 phosphorylation of the armadillo repeat region of beta-catenin potentiates Wnt signaling. J Biol Chem 278: 24018-24025.

18. Wu H, Symes K, Seldin DC, Dominguez I (2009) Threonine 393 of beta-catenin regulates interaction with Axin. J Cell Biochem 108: 52-63.

19. Dominguez I, Mizuno J, Wu H, Imbrie GA, Symes K, et al. (2005) A role for CK2alpha/beta in Xenopus early embryonic development. Mol Cell Biochem 274: 125-131.

20. Schneider S, Steinbeisser H, Warga RM, Hausen P (1996) Beta-catenin translocation into nuclei demarcates the dorsalizing centers in frog and fish embryos. Mech Dev 57: 191-198.

21. Smith RC (1986) Protein synthesis and messenger RNA levels along the animal-vegetal axis during early Xenopus development. J Embryol Exp Morphol 95: 15-35 
Citation: Imbrie GA, Wu H, Seldin DC, Dominguez I (2012) Asymmetric Localization of CK2a During Xenopus Oogenesis . Human Genet Embryol S4:001. doi:10.4172/2161-0436.S4-001

22. Sindelka R, Jonak J, Hands R, Bustin SA, Kubista M (2008) Intracellular expression profiles measured by real-time PCR tomography in the Xenopus laevis oocyte. Nucleic Acids Res 36: 387-392.

23. Moen TL, Namenwirth M (1977) The distribution of soluble proteins along the animal-vegetal axis of frog eggs. Dev Biol 58: 1-10.

24. Hausen P, Riebesell M (1991) The early development of Xenopus laevis. An atlas of the histology. 1991: Springer-Verlag.

25. Denegre JM, Ludwig ER, Mowry KL (1997) Localized maternal proteins in Xenopus revealed by subtractive immunization. Dev Biol 192: 446-454.

26. Ku M, Melton DA (1993) Xwnt-11: a maternally expressed Xenopus wnt gene. Development 119: 1161-1173.

27. Lustig KD, Kroll KL, Sun EE, Kirschner MW (1996) Expression cloning of a Xenopus T-related gene (Xombi) involved in mesodermal patterning and blastopore lip formation. Development 122: 4001-4012.

28. Stennard F, Carnac G, Gurdon JB (1996) The Xenopus T-box gene, Antipodean, encodes a vegetally localised maternal mRNA and can trigger mesoderm formation. Development 122: 4179-4188.

29. Zhang J, King ML (1996) Xenopus VegT RNA is localized to the vegetal cortex during oogenesis and encodes a novel T-box transcription factor involved in mesodermal patterning. Development 122: 4119-4129.

30. Houston DW, Zhang J, Maines JZ, Wasserman SA, King ML (1998) A Xenopus DAZ-like gene encodes an RNA component of germ plasm and is a functional homologue of Drosophila boule. Development 125: 171-180.

31. Rebagliati MR, Weeks DL, Harvey RP, Melton DA (1985) Identification and cloning of localized maternal RNAs from Xenopus eggs. Cell 42: 769-777.

32. Weeks DL, Melton DA (1987) A maternal mRNA localized to the vegetal hemisphere in Xenopus eggs codes for a growth factor related to TGF-beta. Cell 51: 861-867.

33. Schnapp BJ, Arn EA, Deshler JO, Highett MI (1997) RNA localization in Xenopus oocytes. Semin Cell Dev Biol 8: 529-540.

34. King ML, Messitt TJ, Mowry KL (2005) Putting RNAs in the right place at the right time: RNA localization in the frog oocyte. Biol Cell 97: 19-33.

35. Claussen M, Pieler T (2010) Identification of vegetal RNA-localization elements in Xenopus oocytes. Methods 51: 146-151.

36. Whitfield TT, Heasman J, Wylie CC (1995) Early embryonic expression of XLPOU-60, a Xenopus POU-domain protein. Dev Biol 169: 759-769.

37. Cao Y, Siegel D, Donow C, Knochel S, Yuan L, et al. (2007) POU-V factors antagonize maternal VegT activity and beta-Catenin signaling in Xenopus embryos. Embo J 26: 2942-2954.

38. Christian JL, Gavin BJ, McMahon AP, Moon RT (1991) Isolation of cDNAs partially encoding four Xenopus Wnt-1/int-1-related proteins and characterization of their transient expression during embryonic development. Dev Biol 143: 230-234.

39. Ossipova O, He X, Green J (2002) Molecular cloning and developmental expression of Par-1/MARK homologues XPar-1A and XPar-1B from Xenopus laevis. Mech Dev 1: S143-S148.

40. Goto T, Kinoshita T (1999) Maternal transcripts of mitotic checkpoint gene, Xbub3, are accumulated in the animal blastomeres of Xenopus early embryo. DNA Cell Biol 18: 227-234.

41. Hasegawa K, Shiraishi T, Kinoshita T (1999) Xoom: a novel oocyte membrane protein maternally expressed and involved in the gastrulation movement of Xenopus embryos. Int J Dev Biol 43: 479-485

42. Devic E, Paquereau L, Rizzoti K, Monier A, Knibiehler B, et al. (1996) The mRNA encoding a beta subunit of heterotrimeric GTP-binding proteins is localized to the animal pole of Xenopus laevis oocyte and embryos. Mech Dev 59: 141-151.

43. Meyer D, Durliat M, Senan F, Wolff M, Andre M, et al. (1997) Ets-1 and Ets-2 proto-oncogenes exhibit differential and restricted expression patterns during Xenopus laevis oogenesis and embryogenesis. Int J Dev Biol 41: 607-620.

44. Schroeder KE, Yost HJ (1996) Xenopus poly (A) binding protein maternal RNA is localized during oogenesis and associated with large complexes in blastula. Dev Genet 19: 268-276.
45. Hasegawa K, Sakurai N, Kinoshita T (2001) Xoom is maternally stored and functions as a transmembrane protein for gastrulation movement in Xenopus embryos. Dev Growth Differ 43: 25-31.

46. Kume S, Muto A, Aruga J, Nakagawa T, Michikawa T, et al. (1993) The Xenopus IP3 receptor: structure, function, and localization in oocytes and eggs. Cell 73: 555-570.

47. Dumont JN (1972) Oogenesis in Xenopus laevis (Daudin). I. Stages of oocyte development in laboratory maintained animals. J Morphol 136: 153-179.

48. Dominguez I, Marshall MS, Gibbs JB, Garcia de Herreros A, Cornet ME, et al. (1991) Role of GTPase activating protein in mitogenic signalling through phosphatidylcholine-hydrolysing phospholipase C. Embo J 10: 3215-3220.

49. Sindelka R, Sidova M, Svec D, Kubista M (2010) Spatial expression profiles in the Xenopus laevis oocytes measured with qPCR tomography. Methods 51 $87-91$

50. Smith RC, Neff AW, Malacinski GM (1986) Accumulation, organization and deployment of oogenetically derived Xenopus yolk/nonyolk proteins. J Embryol Exp Morphol 97 Suppl: 45-64.

51. Denegre JM, Ludwig ER, Mowry KL (1997) Localized maternal proteins in Xenopus revealed by subtractive immunization. Dev Biol 192: 446-454.

52. Wilhelm V, Rojas P, Gatica M, Allende CC, Allende JE (1995) Expression of the subunits of protein kinase CK2 during oogenesis in Xenopus laevis. Eur $J$ Biochem 232: 671-676.

53. Zhou Y, King ML (1996) Localization of Xcat-2 RNA, a putative germ plasm component, to the mitochondrial cloud in Xenopus stage I oocytes. Development 122: 2947-2953.

54. Kloc M, Larabell C, Etkin LD (1996) Elaboration of the messenger transport organizer pathway for localization of RNA to the vegetal cortex of Xenopus oocytes. Dev Biol 180: 119-130.

55. Sardet C, Prodon F, Pruliere G, Chenevert J (2004) Polarization of eggs and embryos: some common principles. Med Sci 20: 414-423.

56. Heasman J (2006) Maternal determinants of embryonic cell fate. Semin Cell Dev Biol 17: 93-98.

57. Tam PP, Loebel DA (2007) Gene function in mouse embryogenesis: get set for gastrulation. Nat Rev Genet 8: 368-381.

58. Raya A, Belmonte JC (2006) Left-right asymmetry in the vertebrate embryo: from early information to higher-level integration. Nat Rev Genet 7: 283-293.

59. Suzuki A, Ohno S (2006) The PAR-aPKC system: lessons in polarity. J Cell Sci 119: 979-987.

60. Huynh JR, St Johnston D (2004) The origin of asymmetry: early polarisation of the Drosophila germline cyst and oocyte. Curr Biol 14: R438-449.

61. Sardet C, Dru P, Prodon F (2005) Maternal determinants and mRNAs in the cortex of ascidian oocytes, zygotes and embryos. Biol Cell 97: 35-49.

62. White JA, Heasman J (2008) Maternal control of pattern formation in Xenopus laevis. J Exp Zoolog B Mol Dev Evol 310: 73-84.

63. Sardet C, Paix A, Prodon F, Dru P, Chenevert J (2007) From oocyte to 16cell stage: cytoplasmic and cortical reorganizations that pattern the ascidian embryo. Dev Dyn 236: 1716-1731.

64. Nakaya M, Fukui A, Izumi Y, Akimoto K, Asashima M, et al. (2000) Meiotic maturation induces animal-vegetal asymmetric distribution of aPKC and ASIP/ PAR-3 in Xenopus oocytes. Development 127: 5021-5031.

65. Zhang C, Vilk G, Canton DA, Litchfield DW (2002) Phosphorylation regulates the stability of the regulatory CK2beta subunit. Oncogene 21: 3754-3764.

66. Shahbabian K, Chartrand P (2012) Control of cytoplasmic mRNA localization. Cell Mol Life Sci 69: 535-552.

67. Thio GL, Ray RP, Barcelo G, Schupbach T (2000) Localization of gurken RNA in Drosophila oogenesis requires elements in the $5^{\prime}$ and 3 ' regions of the transcript. Dev Biol 221: 435-446.

68. Chartrand $\mathrm{P}$, Meng XH, Singer RH, Long RM (1999) Structural elements required for the localization of $\mathrm{ASH} 1 \mathrm{mRNA}$ and of a green fluorescent protein reporter particle in vivo. Curr Biol 9: 333-336. 
Citation: Imbrie GA, Wu H, Seldin DC, Dominguez I (2012) Asymmetric Localization of CK2 $\alpha$ During Xenopus Oogenesis . Human Genet Embryol S4:001. doi:10.4172/2161-0436.S4-001

Page 9 of 9

69. Washida H, Sugino A, Kaneko S, Crofts N, Sakulsingharoj C, et al. (2009) Identification of cis-localization elements of the maize 10-kDa delta-zein and their use in targeting RNAs to specific cortical endoplasmic reticulum subdomains. Plant J 60: 146-155.
70. St Johnston D (2005) Moving messages: the intracellular localization of mRNAs. Nat Rev Mol Cell Biol 6: 363-375.

This article was originally published in a special issue, Molecular Mechanisms

of Embryogenesis and Tumorigenesis handled by Editor(s). Dr. Jorn Bullerdiek, University of Bremen, Germany 